\title{
DEVELOPING MARKETING STRATEGY BASED ON ENGINEERING MENU AT AGENG RESTAURANT, TEBET DISTRICT, SOUTH JAKARTA
}

\author{
Meri Safarwati PUTRI ${ }^{1}$, and Siti Chairiyah BATUBARA ${ }^{2 *}$ \\ ${ }^{1}$ Communications Science Department, Sahid University, Indonesia \\ ${ }^{2}$ Food Technology Department, Sahid University, Indonesia \\ *siti.chairiyah.batubara@gmail.com
}

\begin{abstract}
Ageng Restaurant is one of the restaurant in Tebet district of South Jakarta that serves many varian menus. A good menu can be seen from the level of sales and ability to provide benefits or the level of popularity and profitability. Therefore, it is necessary to analyze the engineering menu to obtain the right marketing strategy. The method of this research was a case study. The data was analyzed by descriptive analysis which includes analysis of contribution margin and analysis of popularity index, menu classification and SWOT analysis. The Analysis on 53 menu items for 6 months show that in menu's divided into stars' category (13 menu's), plowhorses category (22 menu's), puzzles category (7 menu's) and dog category (11 menu's). Based on category, Ageng's Restaurant develop Marketing strategy. Menu with star classification is a good featured menu. For this group, Ageng Restaurant needs to maintain the quality of the menu, so that it remains an idol for customers. Menus classified as plowhorses can be improved by increasing the selling price. It can be done by innovating so that it can increase the selling price. But do not increase the selling price too high, because it can affect popularity. Menus with puzzles classification can be improved by doing more promotions and give discounts or making it as a recommended menu for customers. Menu with dogs' classification the management needs to improve the menu again in terms of taste, appearance, portion, price, quality and can innovate the menu using the same basic ingredients. The last step that can be done is to delete the menu in this category and replace it with a new menu.
\end{abstract}

Keywords: Popularity Index, Profitability, Menu's Classification, Contribution Margin

\section{BACKGROUND}

Today's tourism industry is an industry that is being promoted by the government in addition to the natural products industry which has an important role in the Indonesian economy. The development paradigm of several countries is now more oriented towards the development of the service and industrial sectors, including the tourism industry. One of the facilities that support tourism activities is a service business that provides food and drinks or commonly referred to as a restaurant. The definition of a restaurant according to Ninemeier and Hayes (2006) is "A food service operation that brings profit whose main basis includes the sale of food and beverages to consumers. individuals and guests in small groups".

One of the factors that become the main strength for the business in this restaurant sector is the menu, even the menu can be also the identity of the restaurant. A good menu can be the main source of income for a restaurant and can be capital for a restaurant to last long enough even for years. Therefore, the ability to determine the menu will determine the success of the culinary business, as stated by Ninemeir (1990), namely "There is a belief in the food service industry that everything starts with the menu". Which means that there is a belief in the food service industry that it all starts with the menu. Even before the kitchen or restaurant was built, a menu list had to be made or thought out in advance.

As an important factor in supporting the success of a restaurant business to sell food and drinks, a menu must be chosen properly, in terms of taste, appearance and attractive prices for consumers and also of course must provide benefits for the restaurant. Apart from being a tool for offering products, in a restaurant business a menu also need to be evaluated. This aims to assist the management in planning the next menu. In addition, with the menu evaluation process, management can find out if there are menus that are less popular, so that management can immediately take action on problems.

A product has a period called the Life Cycle, where a product will experience a period of product development, introduction, growth, maturity which finally cannot develop again and can be forgotten or decline. Based on the graph, then, efforts are needed to maintain the menu so that it does not experience a decline. By monitoring the menu offered, it is hoped that the restaurant business will not be forgotten by customers because it has anticipated the problems of the Life Cycle menu offered. A good menu can be seen from the level of sales and its ability to provide profits or the level of popularity and profitability. As stated by Ninemeir in his book entitled Planning and Control for the Food and Beverage Operation (2009) "There are two factors measure of how to good menu is: its popularity and its profitability". The statement shows that there are 2 (two) benchmarks for a menu that can be said to be good, namely in terms of popularity and in terms of benefits. To see the relationship between the two parameters, it is necessary to make a comparison that can provide conclusions about the position of each type of food in the menu mix offered. So that a category will be obtained about the ability of the food menu item. As stated by Ninemeir (1991:106) that, "with these tools of menu engineering manager can evaluate profitability of menu items and classify them as either stars, plowhorse, puzzles or dogs". Which can be interpreted by menu engineering a manager can evaluate the benefits and classify the menu into groups of stars, plowhorses, puzzles or dogs. This study aims to obtain Marketing Strategy at Ageng Restaurant based on Menu Engineering. 


\section{METHODS}

The research method used in this research is descriptive method that it is not intended to test certain hypotheses, but only describes "what is about something (Sugiyono, 2009). Interviews were conducted with the Manager of Rumah Makan Ageng to obtain information in the form of the history of the establishment of Rumah Makan Ageng, organizational structure, number of employees, seating capacity, facilities, as well as variable ways, symptoms and how Rumah Makan Ageng evaluates the menu. the documentation data collected was data in the form of photographs of activities at RM Ageng. There are several steps in determining the analysis into the engineering menu-based marketing development strategy at Rumah Makan Ageng as follows:

1. Determination of Menu Popularity Index

Menu evaluation can be done by analyzing the level of popularity and the level of profit obtained by the company from each product sold. The popularity index can be calculated by dividing the number of servings sold from one type of menu by the total servings sold from all menus.

$$
\begin{aligned}
& \text { Popularity Index } \\
& \qquad=\frac{100 \%}{\text { Number of menu item }} \times 70 \%
\end{aligned}
$$

2. Determination of The Mix Percentage Menu

Menu mix percentage is the percentage of total sales from each menu, which is obtained by dividing the number of servings sold from each menu by the total of all menus sold and then multiplied by 100 .

$$
\begin{aligned}
\text { Menu Mix } \% & \\
= & \text { Number of item Sold } \\
& \times 100 \%
\end{aligned}
$$

\section{Etermination of Contribution Margin}

Contribution margin is the data used to determine the level of profitability of a menu. Which can be interpreted $\mathrm{CM}$ is calculated by subtracting the cost of food from the total income.

$$
\begin{aligned}
& \text { Contribution margin } \\
& \quad=\text { selling price } \\
& \quad-\text { food cost per portion }
\end{aligned}
$$

4. Etermination of Average Contribution Margin

The average contribution margin is obtained by dividing the total contribution margin by the total menu sold. Thus, it can be formulated as follows: The result of the Item Contribution Margin will be compared with the Average Contribution Margin to see how high or low the contribution level of a menu item is.

$$
\begin{aligned}
& \text { Average contribution margin } \\
& \qquad=\frac{\text { Total contribution margin }}{\text { Total number of item sold }}
\end{aligned}
$$

\section{Determination of Menu Classification}

The menu can be evaluated by analyzing the popularity and ability of each type of food and drink on a menu in contributing to profits. Each menu is classified into four categories, namely Stars, Plowhorses, Puzzles, and Dogs.

\section{RESULTS AND DISCUSSION \\ Menu Popularity Index}

The popularity of a menu can be known based on its

\begin{tabular}{|c|c|c|c|c|c|c|c|c|}
\hline \multirow{2}{*}{ No } & \multirow{2}{*}{ Food Type } & \multicolumn{4}{|c|}{ Sales Amount, Year 2019} & \multicolumn{3}{|c|}{ Sales Amount, Year 2020} \\
\hline & & Sept & Oct & Nop & Des & Jan & Feb & Total \\
\hline \multirow[t]{8}{*}{1.} & Main Course & & & & & & & \\
\hline & White rice & 1250 & 1248 & 1249 & 1239 & 1245 & 1249 & 7480 \\
\hline & White rice $1 / 2$ portion & 500 & 425 & 438 & 413 & 450 & 467 & 2693 \\
\hline & Fried Chicken & 125 & 123 & 125 & 124 & 123 & 125 & 745 \\
\hline & Grilled Chicken & 124 & 125 & 124 & 125 & 123 & 124 & 745 \\
\hline & Penyet Chicken & 123 & 125 & 123 & 125 & 124 & 123 & 743 \\
\hline & Javanese Noodles / Nyemek Noodles & 100 & 95 & 93 & 89 & 90 & 94 & 561 \\
\hline & Sub-Total & & & & & & & 12967 \\
\hline \multirow[t]{5}{*}{2.} & Yellow Soto and Gurame Soup & & & & & & & \\
\hline & Chicken Soto & 350 & 363 & 361 & 365 & 370 & 373 & 2182 \\
\hline & Meat Soto & 475 & 489 & 488 & 485 & 500 & 497 & 2934 \\
\hline & Soup and Sour Gurame Spicy & 147 & 145 & 148 & 146 & 149 & 147 & 882 \\
\hline & Sub Total & & & & & & & 5998 \\
\hline \multirow[t]{5}{*}{3.} & Fried noodles & & & & & & & \\
\hline & Special fried noodle & 375 & 369 & 373 & 368 & 372 & 369 & 2226 \\
\hline & Chicken Fried Noodles & 245 & 249 & 247 & 244 & 250 & 247 & 1482 \\
\hline & Egg Fried Noodles & 371 & 369 & 373 & 368 & 371 & 358 & 2210 \\
\hline & Sub Total & & & & & & & 5918 \\
\hline \multirow[t]{5}{*}{4.} & Fried rice & & & & & & & \\
\hline & Special fried rice & 250 & 245 & 250 & 247 & 242 & 245 & 1479 \\
\hline & Chicken fried rice & 73 & 73 & 72 & 75 & 70 & 72 & 435 \\
\hline & Fried rice with egg & 372 & 369 & 373 & 368 & 369 & 373 & 2224 \\
\hline & Thai Fried Rice & 49 & 42 & 46 & 43 & 47 & 44 & 271 \\
\hline
\end{tabular}
popularity index. This can be analyzed based on the number of sales from the menu offered. Sales history is a format for recapitulating sales over a period. The Sales History of Food Products at Ageng Restaurant for the September 2019 - February 2020 period can be seen in Table 1.

Tabel 1. Sales History of Food Products at Ageng Restaurant for the September 2019 Period - February 2020 


\begin{tabular}{|c|c|c|c|c|c|c|c|c|}
\hline \multirow{2}{*}{ No } & \multirow{2}{*}{ Food Type } & \multicolumn{4}{|c|}{ Sales Amount, Year 2019} & \multicolumn{3}{|c|}{ Sales Amount, Year 2020} \\
\hline & & Sept & Oct & Nop & Des & Jan & Feb & Total \\
\hline & Ageng Fried Rice & 75 & 72 & 74 & 71 & 70 & 73 & 435 \\
\hline & Sub Total & & & & & & & 4844 \\
\hline \multirow[t]{8}{*}{5.} & Complementary Menu & & & & & & & \\
\hline & Lalap + Sambel & 49 & 48 & 43 & 41 & 42 & 38 & 261 \\
\hline & Sayur Asem & 100 & 95 & 85 & 90 & 98 & 101 & 569 \\
\hline & Urap & 72 & 75 & 73 & 73 & 74 & 71 & 438 \\
\hline & Egg Tofu & 369 & 373 & 368 & 371 & 369 & 375 & 2225 \\
\hline & Tempe penyet & 250 & 247 & 242 & 245 & 249 & 247 & 1480 \\
\hline & Tahu/Tempe Goreng & 245 & 249 & 247 & 244 & 242 & 245 & 1472 \\
\hline & Sub Total & & & & & & & 6445 \\
\hline \multirow[t]{4}{*}{6.} & Oriental Food & & & & & & & \\
\hline & Capcay & 95 & 85 & 90 & 98 & 101 & 89 & 558 \\
\hline & Capcay Rice & 75 & 72 & 75 & 73 & 74 & 71 & 440 \\
\hline & Sub Total & & & & & & & 998 \\
\hline \multirow[t]{5}{*}{7.} & Fried vermicelli & & & & & & & \\
\hline & Special Fried Vermicelli & 125 & 123 & 125 & 123 & 125 & 124 & 745 \\
\hline & Chicken Fried Vermicelli & 70 & 72 & 75 & 73 & 74 & 71 & 435 \\
\hline & Egg Fried Vermicelli & 247 & 250 & 245 & 249 & 247 & 244 & 1482 \\
\hline & Sub Total & & & & & & & 2662 \\
\hline \multirow[t]{5}{*}{8.} & Fried Kwetiau & & & & & & & \\
\hline & Special Fried Kwetiau & 123 & 118 & 124 & 122 & 123 & 121 & 731 \\
\hline & Chicken Fried Kwetiau & 49 & 55 & 50 & 48 & 51 & 45 & 298 \\
\hline & Egg Fried Kwetiau & 247 & 242 & 245 & 249 & 247 & 244 & 1474 \\
\hline & Sub Total & & & & & & & 2503 \\
\hline \multirow[t]{12}{*}{9.} & Drink & & & & & & & \\
\hline & Fresh Tea & 124 & 123 & 125 & 123 & 125 & 124 & 744 \\
\hline & Sweet Tea & 371 & 369 & 373 & 368 & 370 & 369 & 2220 \\
\hline & Fresh Ice Tea & 488 & 473 & 475 & 478 & 490 & 491 & 2895 \\
\hline & Sweet Ice Tea & 492 & 455 & 473 & 475 & 478 & 490 & 2863 \\
\hline & Orenge Juice & 366 & 370 & 372 & 369 & 373 & 368 & 2218 \\
\hline & Lime Ice & 75 & 72 & 74 & 71 & 70 & 73 & 435 \\
\hline & Dark Coffee & 250 & 242 & 245 & 250 & 247 & 244 & 1478 \\
\hline & Mix Coffee & 124 & 117 & 124 & 122 & 123 & 121 & 731 \\
\hline & Milk Kpoi & 72 & 74 & 71 & 70 & 73 & 75 & 435 \\
\hline & Aqua $600 \mathrm{ml}$ & 247 & 244 & 242 & 245 & 250 & 247 & 1475 \\
\hline & Sub Total & & & & & & & 15494 \\
\hline \multirow[t]{9}{*}{10.} & Saving package & & & & & & & \\
\hline & Package 1 (Rice, Fr. Chicken, Aqua Cup) & 123 & 120 & 117 & 124 & 121 & 124 & 729 \\
\hline & Package 2 Rice, Fr.Chicken,S.Asem, Aqua Cup) & 124 & 121 & 124 & 118 & 121 & 123 & 731 \\
\hline & Package 3 (Rice, Fr. Chicken,Urap, Aqua Cup) & 125 & 121 & 120 & 122 & 119 & 124 & 731 \\
\hline & Package 4 (Rice, Tofu, Tempe, S. Asem, Aqua Cup) & 121 & 125 & 121 & 123 & 119 & 120 & 729 \\
\hline & Package 5 (Rice, Tofu, Tempe,Urap, Aqua Cup) & 125 & 121 & 120 & 118 & 121 & 123 & 728 \\
\hline & Package 6 (Rice, Grilled Chick,S. Asem, Aqua Cup) & 123 & 117 & 124 & 122 & 123 & 121 & 730 \\
\hline & Package 7 (Rice, Grilled Chick, Urap, Aqua Cup) & 125 & 123 & 119 & 120 & 124 & 125 & 736 \\
\hline & Sub Total & & & & & & & 5114 \\
\hline \multirow[t]{7}{*}{11.} & Serbu Pack & & & & & & & \\
\hline & Serbu 1 (Fried Rice, Aqua Cup) & 123 & 121 & 120 & 118 & 121 & 123 & 726 \\
\hline & Serbu 2 (Fried Noodles, Aqua Cup) & 244 & 242 & 245 & 250 & 247 & 246 & 1474 \\
\hline & Serbu 3 (Fried Kwetiau, Aqua Cup) & 123 & 123 & 117 & 124 & 122 & 123 & 732 \\
\hline & Serbu 4 (Fried Vermicelli, Aqua Cup) & 125 & 121 & 124 & 118 & 121 & 123 & 732 \\
\hline & Sub Total & & & & & & & 3664 \\
\hline & Total Sales Amount & & & & & & & 66607 \\
\hline
\end{tabular}

The popularity level can be found by dividing $100 \%$ by the total calculation menu in each category, then the result of the calculation is multiplied by $70 \%$, which will get the category popularity index. This popularity index is the limit to determine whether the menu in that category is popular or less popular. If the calculation result of menu mix $\%$ of a menu in that category exceeds its popularity index, the menu can be classified as popular, but if it is lower then the menu is categorized as less popular. Based on the data obtained, it is known that the Menu Popularity Index at Rumah Makan Ageng for the September 2019 February 2020 period can be seen in Table 2 .

\section{Menu Popularity Index}

After Popularity Index was found, then its need to determined the Mix menu category of each menu. Menu Category can devided into high and low category. 
Tabel 2. Menu Popularity Index at Ageng Restaurant Period September 2019 - February 2020

\begin{tabular}{clcc}
\hline No & \multicolumn{1}{c}{ Menu Category } & $\begin{array}{c}\text { Number of } \\
\text { Items }\end{array}$ & $\begin{array}{c}\text { Popularity } \\
\text { Index }\end{array}$ \\
\hline 1 & Main course & 6 & $12 \%$ \\
2. & Yellow Soto and Gurame Soup & 3 & $23 \%$ \\
3. & Fried Noodles & 3 & $23 \%$ \\
4. & Fried Rice & 6 & $12 \%$ \\
5. & Complementary Menu & 6 & $12 \%$ \\
6. & Oriental Food & 2 & $35 \%$ \\
7. & Fried vermicelli & 3 & $23 \%$ \\
8. & Fried Kwetiau & 3 & $23 \%$ \\
9. & Drink & 10 & $7 \%$ \\
10. & Saving Packet & 7 & $10 \%$ \\
11 & Serbu Packet & 4 & $18 \%$ \\
& Total Menu & 53 & \\
\hline
\end{tabular}

\section{Contribution Margin at Ageng Restaurant}

Contribution margin is the data used to determine the level of profitability of a menu. There are two determining factors in the contribution margin, namely food cost and selling price. After calculating the contribution margin of each menu, the next step is to calculate the average contribution margin of each menu category. If the contribution margin of an item is lower than the average contribution margin, it can be recorded or marked as low, otherwise if the contribution margin of an item is higher than the average contribution margin, it can be marked as high (high).

\section{Menu Classification at Ageng Restaurant}

The menu can be evaluated by analyzing the popularity and ability of each type of food and drink on a menu in contributing to profits. After a menu has been known the level of popularity and profitability, then a menu can be entered into a worksheet called a menu engineering worksheet. The example of calculation to classify menu at Ageng Restaurant can be seen in Table 3. The complete data of menu classification shown at Table 4.

Tabel 3. The Example of calculation to Classification Menu at Ageng Restaurant

\begin{tabular}{|c|c|c|c|c|c|c|c|c|c|c|c|c|}
\hline \multirow{3}{*}{ No } & $\mathbf{A}$ & B & C & D & $\mathbf{E}$ & $\mathbf{F}$ & G & H & I & $\mathbf{J}$ & $\mathbf{K}$ & $\mathbf{L}$ \\
\hline & $\begin{array}{l}\text { Menu Item } \\
\text { Name }\end{array}$ & $\begin{array}{l}\text { Number } \\
\text { Sold }\end{array}$ & $\begin{array}{c}\text { Menu } \\
\text { Mix } \\
\%\end{array}$ & $\begin{array}{l}\text { Food } \\
\text { Cost }\end{array}$ & $\begin{array}{l}\text { Sell } \\
\text { Price }\end{array}$ & $\begin{array}{l}\text { Item } \\
\mathrm{CM}\end{array}$ & $\begin{array}{c}\text { Menu } \\
\text { Cost }\end{array}$ & $\begin{array}{c}\text { Menu } \\
\text { Revenue }\end{array}$ & $\begin{array}{l}\text { Total } \\
\text { Profit }\end{array}$ & $\begin{array}{c}\mathrm{CM} \\
\text { Category }\end{array}$ & $\begin{array}{c}\text { M.M } \\
\text { Category }\end{array}$ & $\begin{array}{l}\text { Menu Item } \\
\text { Class }\end{array}$ \\
\hline & Rice & & & & & $(E-D)$ & $(D * B)$ & $(E * B)$ & $(H-G)$ & & & \\
\hline 1 & White Rice & 7480 & $58 \%$ & 2000 & 5000 & 3000 & 14960000 & 37400000 & 22440000 & Low & High & Plowhorse \\
\hline 2 & $\begin{array}{l}\text { White Rice } 1 / 2 \\
\text { portion }\end{array}$ & 2693 & $21 \%$ & 1500 & 3000 & 1500 & 4039500 & 8079000 & 4039500 & Low & High & Plowhorse \\
\hline 3 & $\begin{array}{l}\text { Fried Chicken } \\
\text { Grilled }\end{array}$ & 745 & $6 \%$ & 11000 & 17000 & 6000 & 8195000 & 12665000 & 4470000 & High & Low & Puzzle \\
\hline 4 & Chicken & 745 & $6 \%$ & 12000 & 18000 & 6000 & 8940000 & 13410000 & 4470000 & High & Low & Puzzle \\
\hline 5 & Penyet Chicken & 743 & $6 \%$ & 12000 & 18000 & 6000 & 8916000 & 13374000 & 4458000 & High & Low & Puzzle \\
\hline \multirow[t]{6}{*}{6} & $\begin{array}{l}\text { Javanese } \\
\text { noodles/ } \\
\text { Nyemek } \\
\text { noodles }\end{array}$ & 561 & & & & & 6732000 & 11781000 & 5049000 & & & \\
\hline & Totals & 12967 & \multirow{2}{*}{$4 \%$} & \multirow{2}{*}{12000} & \multirow{2}{*}{21000} & \multirow{2}{*}{9000} & 51782500 & 96709000 & 44926500 & \multirow{2}{*}{ High } & \multirow{2}{*}{ Low } & \multirow{2}{*}{ Puzzle } \\
\hline & $\begin{array}{l}\text { Totals Menu } \\
\text { item }\end{array}$ & 6 & & & & & & & & & & \\
\hline & \multicolumn{2}{|c|}{ Percentage Menu Mix } & $12 \%$ & & & & & & & & & \\
\hline & Menu Food Cost & & & & & & $54 \%$ & & & & & \\
\hline & Average CM & & & & & & & & 5250 & & & \\
\hline
\end{tabular}

Tabel 4. Classification Menu

\begin{tabular}{|c|c|c|c|c|c|c|c|c|c|c|c|c|}
\hline \multirow{3}{*}{ No } & $\mathbf{A}$ & B & C & D & $\mathbf{E}$ & $\mathbf{F}$ & G & H & I & J & $\mathbf{K}$ & $\mathbf{L}$ \\
\hline & \multirow[t]{2}{*}{ Menu Item Name } & $\begin{array}{l}\text { Number } \\
\text { Sold }\end{array}$ & $\begin{array}{c}\text { Menu } \\
\text { Mix } \\
\%\end{array}$ & $\begin{array}{l}\text { Food } \\
\text { Cost }\end{array}$ & $\begin{array}{l}\text { Sell } \\
\text { Price }\end{array}$ & $\begin{array}{l}\text { Item } \\
\mathrm{CM}\end{array}$ & $\begin{array}{c}\text { Menu } \\
\text { Cost }\end{array}$ & $\begin{array}{c}\text { Menu } \\
\text { Revenue }\end{array}$ & $\begin{array}{l}\text { Total } \\
\text { Profit }\end{array}$ & $\begin{array}{c}\text { CM } \\
\text { Category }\end{array}$ & $\begin{array}{c}\text { M.M } \\
\text { Category }\end{array}$ & $\begin{array}{c}\text { Menu Item } \\
\text { Class }\end{array}$ \\
\hline & & & & & & $(E-D)$ & $(D * B)$ & $(E * B)$ & $(H-G)$ & & & \\
\hline 1 & White Rice & 7480 & $58 \%$ & 2000 & 5000 & 3000 & 14960000 & 37400000 & 22440000 & Low & High & Plowhorse \\
\hline 2 & $\begin{array}{l}\text { White Rice } 1 / 2 \\
\text { portion }\end{array}$ & 2693 & $21 \%$ & 1500 & 3000 & 1500 & 4039500 & 8079000 & 4039500 & Low & High & Plowhorse \\
\hline 3 & Fried Chicken & 745 & $6 \%$ & 11000 & 17000 & 6000 & 8195000 & 12665000 & 4470000 & High & Low & Puzzle \\
\hline 4 & Grilled Chicken & 745 & $6 \%$ & 12000 & 18000 & 6000 & 8940000 & 13410000 & 4470000 & High & Low & Puzzle \\
\hline 5 & Penyet Chicken & 743 & $6 \%$ & 12000 & 18000 & 6000 & 8916000 & 13374000 & 4458000 & High & Low & Puzzle \\
\hline 6 & $\begin{array}{l}\text { Javanese noodles/ } \\
\text { Nyemek noodles }\end{array}$ & 561 & $4 \%$ & 12000 & 21000 & 9000 & 6732000 & 11781000 & 5049000 & High & Low & Puzzle \\
\hline
\end{tabular}




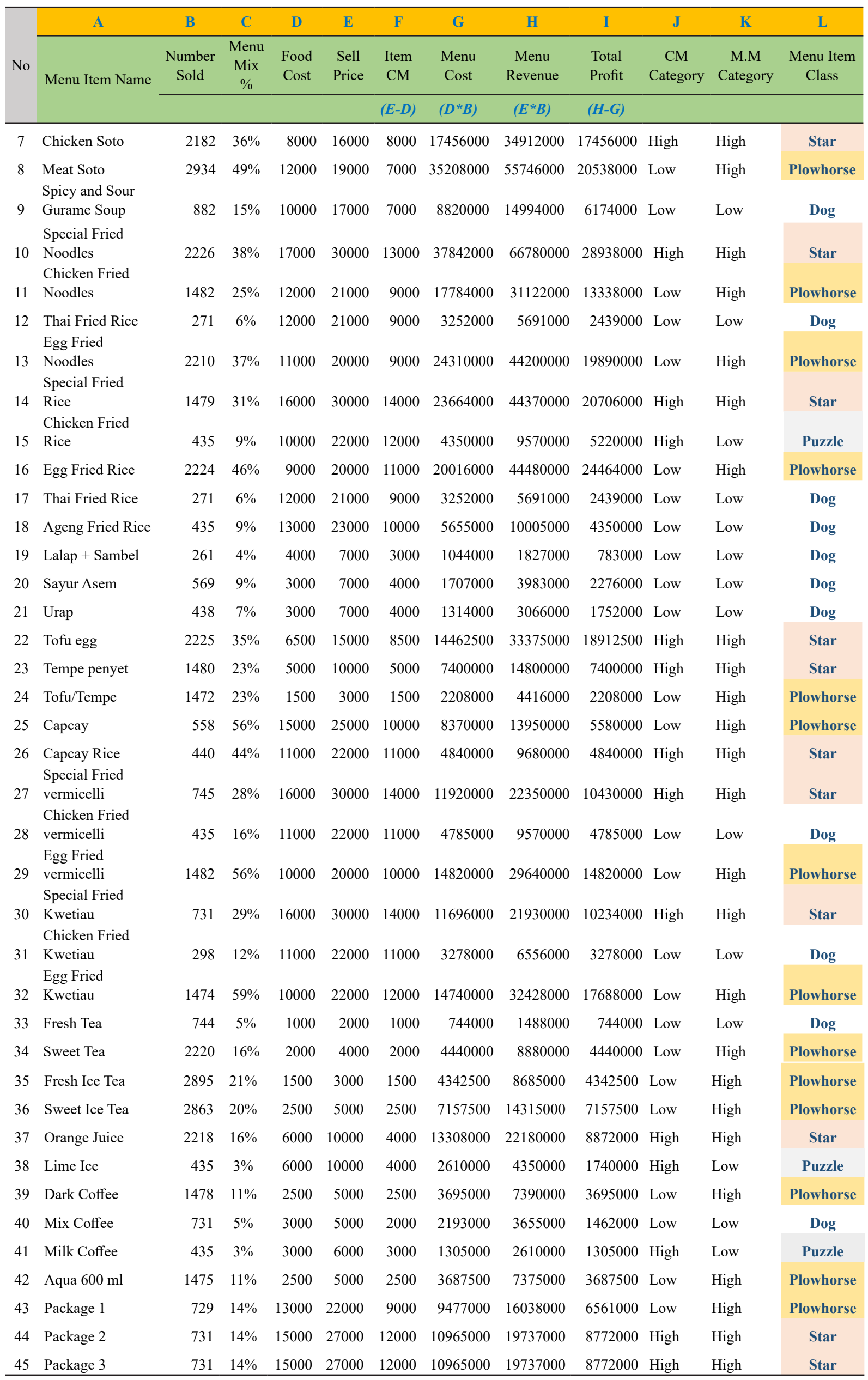




\begin{tabular}{llrrrrrrrrrrrrrrrr}
\hline 46 & Package 4 & 729 & $14 \%$ & 9000 & 17000 & 8000 & 6561000 & 12393000 & 5832000 & Low & High & Plowhorse \\
47 & Package 5 & 728 & $14 \%$ & 9000 & 17000 & 8000 & 6552000 & 12376000 & 5824000 & Low & High & Plowhorse \\
48 & Package 6 & 730 & $14 \%$ & 16000 & 28000 & 12000 & 11680000 & 20440000 & 8760000 & High & High & Star \\
49 & Package 7 & 736 & $14 \%$ & 16000 & 28000 & 12000 & 11776000 & 20608000 & 8832000 & High & High & Star \\
50 & Serbu 1 & 726 & $20 \%$ & 10000 & 18000 & 8000 & 7260000 & 13068000 & 5808000 & Low & High & Plowhorse \\
51 & Serbu 2 & 1474 & $40 \%$ & 10000 & 18000 & 8000 & 14740000 & 26532000 & 11792000 & Low & High & Plowhorse \\
52 & Serbu 3 & 732 & $20 \%$ & 10000 & 18000 & 8000 & 7320000 & 13176000 & 5856000 & Low & High & Plowhorse \\
53 & Serbu 4 & 732 & $20 \%$ & 10000 & 18000 & 8000 & 7320000 & 13176000 & 5856000 & Low & High & Plowhorse \\
\hline
\end{tabular}

\section{CONCLUSIONS}

Based on the results of this study concluded the following conclusions:

1. A total of 32 menu items or $60.38 \%$ are included into the category that has a high popularity index. As many as 21 items (39.62\%) fall into the category that has a low popularity index.

2. A total of 20 menus (38\%) are included into the category with high contribution margin. The other 33 menus (62\%) fall into the category with low contribution margin.

3. Classification of 53 Menu shows:

a. as many as 32 menus $(24,5 \%)$ included into the star category, namely: Chicken soto, Special Fried Noodle, Special Fried vermicelli, Egg tofu, Capcay rice, Special Fried Kwetiau, orange juice, saving packages 2, 3, 6 and 7,

b. as many as 22 menus $(41,5 \%)$ included into the plowhorses category, namely rice, meat soto, $\mathrm{MG}$ chicken, Egg Fried Noodle, Egg Fried Rice, Tofu/ tempe, Capcay, Egg Fried vermicelli, Eggs Fried Kwetiau, Ice tea, Sweet tea,Sweet tea ice, Black coffee, Aqua $600 \mathrm{ml}$, packages 1, 4, and 5, Serbu 1, 2,3 and 4 .

c. as many as 7 menus $(13,2 \%)$ are included into the puzzles category, namely: fried chicken, grilled chicken, chicken penyet, javanese noodles, chicken fried rice, lime ice, coffee milk.

d. as many as 11 menus $(20,8 \%)$ include into the dogs category, namely spicy sour gurame soup, Thai fried rice, Ageng fried rice, urap, chicken fried vermicelli, chicken kwetiau, unsalted tea and mixed coffee.

4. Based on Engineering Menu, Ageng Restaurant can be developing the following marketing strategy:

a. Menus with star classification (high popularity and high profitability) is a good featured menu. For this group, Ageng's Restaurant needs to maintain the quality of the menu, so that it remains an idol for customers.

b. Menus with plowhorses classification (high popularity but low profitability) can be improved by increasing the selling price. It can be done by innovating so that it can increase the selling price. But do not increase the selling price too high, because it can affect popularity.

c. Menus with puzzles classification (low popularity but high profitability) can be improved by doing more promotions for this menu group and discounts or making it as a recommended menu for customers.

d. Menu with dogs classification (low popularity and profitability) the management needs to improve the menu again in terms of taste, appearance, portion, price and quality and can innovate the menu using the same basic ingredients. The last step that can be done is to delete the menu in this category and replace it with a new menu.

This study suggest that the Ageng's restaurant management should evaluate the menu regularlyby using menu engineering, which is every 6 months, so that it can improve its sales performance. Thus, it can be seen to what extent the menu is still desired by customer.

\section{REFERENCES}

Coltman, Michael M dan Martin G. Jagels. 2001. Hospitality Management Accounting, seventh edition. New York.

Dittmer, Paul R. 2003. Principles of Food, Beverage, and Labor Cost Control 7th ed. New York : John Wiley \& Sons.

Dittmer, Paul R, J. Desmond Keefe. 2009. Principle of Food, Beverage, and Labor Cost Control. Hoboken New Jersey: Jhon Wiley \& Sons.

Jagles, Martin G. Ralston, Catherine E. 2007. Hospitality Management Accounting. New Jersey : John Wiley $\&$ Sons.

Miller, Jack E, David R. Hayes dan Lea R. Dopson. 2002. Food and BeverageCost Control. New York, John Wiley \& Sons.

Nawawi, Hadari. 2005. Social Field Research Method. Gadjah Mada University Press.

Ninemeir, Jack D. 1990. Management of Food and Beverage Operation. Michigan. The Educational Intitute of AHMA.

Ninemeier, Jack D. 2009. Planning and Control for Food and Beverage Operation. Michigan : The Educational Institute of AHMA.

Scanlon, Nancy L. 1992. Catering Menu Management. New York. Van Nostand Reinhold. 
Scanlon, Nancy Loman. 2000. Catering Management. USA : John Wiley \& Sons

Schmidgall, Raymond S. 1997. Managerial Accounting. Michigan. The Educational Institute of American Hotel and Motel Association (AHMA).

Siagian, Sondang P. 1992. Managerial Functions. Jakarta: Bina Aksara.
Spears, Gregoire Marry B. 2004. Food ServiceOrganizations 5th ed. New Jersey: Pearson Education.

Sugiyono. 2013. Administration Research Method. Jakarta: Alfabeta.

Sulaiman, Samsudin. 2013. Research Methodology. Bandung : Enhai Press. 